\title{
Clinical experience with a multifunctional, flexible surgery system for endolumenal, single-port, and NOTES procedures
}

\author{
Santiago Horgan · Kari Thompson • Mark Talamini • \\ Alberto Ferreres • Garth Jacobsen • Georg Spaun • \\ John Cullen • Lee Swanstrom
}

Received: 28 August 2009/Accepted: 20 January 2010/Published online: 24 August 2010

(C) The Author(s) 2010. This article is published with open access at Springerlink.com

\begin{abstract}
Background Single-port and incisionless surgical approaches hold the promise of fewer complications, reduced pain, faster recovery, and improved cosmesis compared with traditional open or laparoscopic approaches. The ability to select an access approach (i.e., endolumenal, single-port, transvaginal, or transgastric) with one platform may be important to optimization of individual patient results. The authors report their results using these four separate surgical approaches tailored to three different therapeutic procedures, all with the use of a single flexible platform, the Incisionless Operating Platform (IOP).

Methods After institutional review board approval, the IOP was used to perform nine cholecystectomies via transvaginal (TV) $(n=4)$, transgastric (TG) $(n=4)$, and single-port transumbilical (TU) $(n=1)$ access. Two appendectomies were performed via TG access. Endolumenal access was used for 18 gastric pouch and stoma reductions after Roux-en-Y gastric bypass. The TG and TV procedures involved the use of one to three trocars.
\end{abstract}

Presented at the SAGES 2009 Annual Meeting, April 22-24, 2009, Phoenix, AZ.

S. Horgan $(\bowtie) \cdot$ K. Thompson · M. Talamini · G. Jacobsen ·

J. Cullen

Department of Surgery, University of California, San Diego, 200 West Arbor Drive, 8402 San Diego, CA, USA

e-mail: shorgan@ucsd.edu

\section{A. Ferreres}

Department of General Surgery, Hospital Bocalandro,

University of Buenos Aires, Buenos Aires, Argentina

G. Spaun · L. Swanstrom

Department of Minimally Invasive Surgery, Legacy Health

System, Portland, OR, USA
The recorded data included safety, procedural success, operative time, patient pain assessment (on a 0-10 scale) at discharge, and length of hospital stay.

Results Procedural success was achieved for 16 of 18 endolumenal procedures, 1 of 1 single-port procedure, and 10 of 10 NOTES procedures. For 5 of 10 NOTES procedures, only one small trocar was required. The mean operative times were $79 \mathrm{~min}$ for pouch with stoma reduction, $171 \mathrm{~min}$ for cholecystectomy, and $274 \mathrm{~min}$ for appendectomy. Of 29 patients, 27 were discharged in $24 \mathrm{~h}$ or less. The average pain scores were 0.44 for pouch with stoma reduction, 1.3 for cholecystectomy, and 2.5 for appendectomy. No significant complications occurred. The ergonomics of IOP allowed the surgeon to interface with the system using an endoscopic or laparoscopic orientation. Conclusion Availability of a multifunctional, flexible surgery platform provides a choice of a single-port or incisionless surgical approach with the potential to reduce complications, pain, and recovery time while improving cosmesis.

Keywords Endolumenal surgery - Incisionless surgery · NOTES instrumentation - NOTES surgery ·

Single-port-access surgery

Optimizing access to the surgical site while minimizing trauma to the patient is critical to superior procedural outcomes. The advent of technology designed around a laparoscopic, "less invasive" approach has significantly reduced procedural complication rates, adhesions, scarring, and patient recovery times for many surgical interventions $[1,2]$.

One of the many challenges in natural orifice translumenal surgery (NOTES) has been the lack of adequate 
and innovative technology. Conventional laparoscopic access devices give the surgeon the flexibility to place any number of ports in the optimal orientation for the procedure to be preformed. However, working with an incisionless operative platform that is access neutral has distinct advantages including greater flexibility in the choice of access routes and minimization of surgical impact on the patient.

We report on our clinical work with the Incisionless Operating Platform (IOP) (USGI Medical, San Clemente, CA, USA) to perform several procedures using different natural orifice or single-port approaches.

\section{Materials and methods}

Materials

The 27 procedures performed in this series including transvaginal cholecystectomy $(n=4)$, transgastric cholecystectomy $(n=4)$, transgastric appendectomy $(n=2)$, transumbilical cholecystectomy $(n=1)$, and endolumenal pouch and stoma reduction $(n=16)$ (Table 1$)$. The IOP was used for all the cases. The IOP consists of the Transport (USGI Medical, San Clemente, CA, USA), a flexible, steerable, multilumen access device for passage of instrumentation (Fig. 1), together with an endoscopic grasper and tissue approximation device, the g-Prox (USGI Medical) (Figs. 2 and 3), when approximation is indicated.

The Transport combines many features of a standard endoscope and a laparoscopic trocar. It has four large operating lumens $(6,6,4$, and $4 \mathrm{~mm})$ for insertion of the $\mathrm{g}$-Prox for tissue grasping and approximation, an endoscope (Olympus GIF N-180; Olympus America, Inc., Center Valley, PA, USA, used for this study) for visualization, and various endoscopic instruments as necessary for the procedure.

Insufflation for maintenance of pneumoperitoneum or pneumogastrum was delivered from a standard, high-flow carbon dioxide insufflator connected to a Luer-Lok on the Transport handle. A laparoscopic port was used to monitor intraabdominal pressure in the NOTES cases.

Table 1 Attempted and successful procedures

\begin{tabular}{lll}
\hline Procedure & Access & $\begin{array}{l}\text { No. attempted/ } \\
\text { no. successful }\end{array}$ \\
\hline Cholecystectomy & Transvaginal (TV) & $4 / 4$ \\
& Transgastric (TG) & $4 / 4$ \\
& Transumbilical (TU) & $1 / 1$ \\
Appendectomy & Transgastric (TG) & $2 / 2$ \\
Pouch and stoma reduction & Endolumenal (E) & $16 / 18$ \\
Total & & $27 / 29$ \\
\hline
\end{tabular}

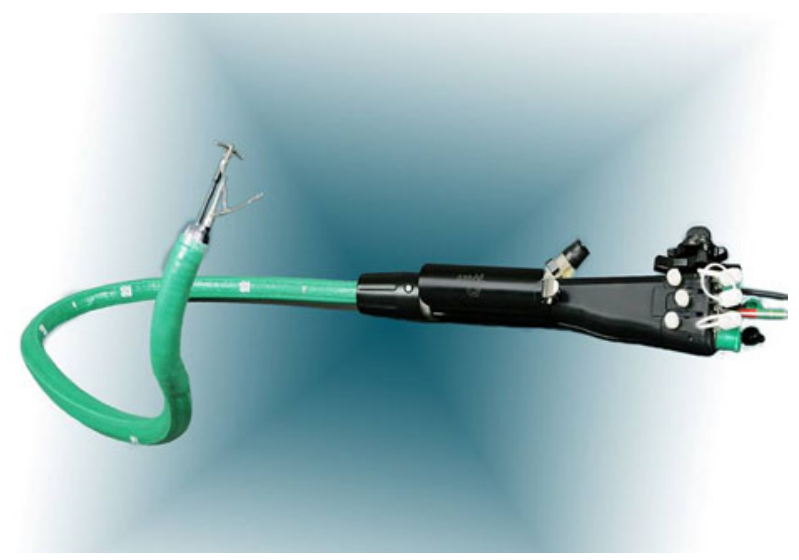

Fig. 1 The Incisionless Operating Platform (IOP) transport device

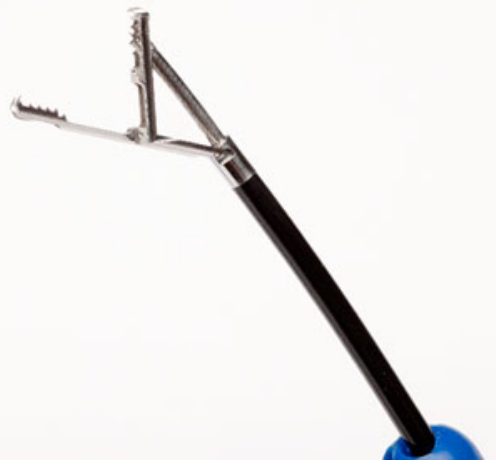

Fig. 2 g-Prox with jaws open

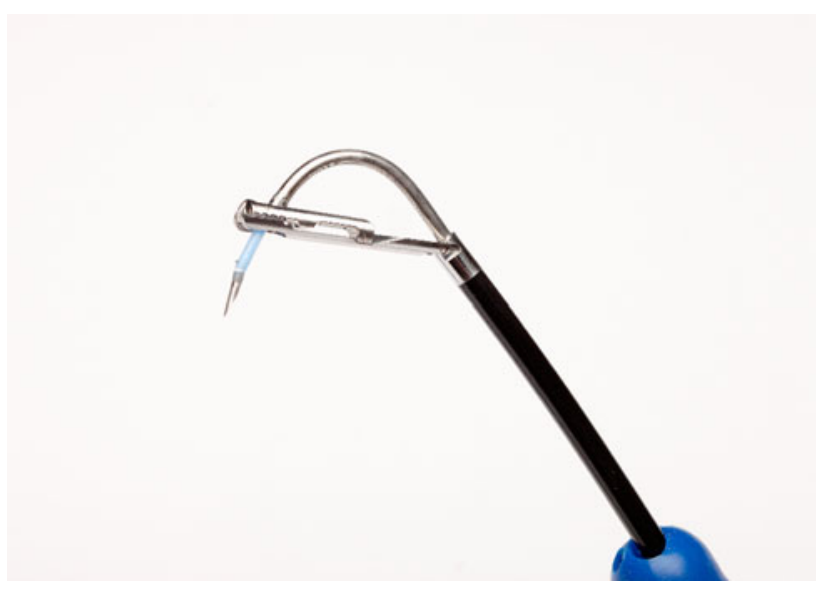

Fig. 3 g-Prox with needle deployed

Methods

All cases were managed at the University of California San Diego (UCSD), San Diego, CA, USA; Legacy Health System, Portland, OR, USA; or Hospital Bocalandro, 
Table 2 Operative times and number of ports used in each case

\begin{tabular}{llll}
\hline Procedure & Access & No. of ports & $\begin{array}{c}\text { Mean operative } \\
\text { time (min) }\end{array}$ \\
\hline Cholecystectomy & Transgastric $(n=4)$ & $2,1,3,2$ & 171 \\
& Transvaginal $(n=4)$ & $2,1,1,1$ & \\
& Transumbilical $(n=1)$ & 1 & \\
Appendectomy & Transgastric $(n=2)$ & 1,2 & 274 \\
Pouch and stoma reduction & Endolumenal $(n=16)$ & & 79
\end{tabular}

University of Buenos Aires, Buenos Aries, Argentina between January 2008 and November of 2008. The transgastric appendectomy and transvaginal cholecystectomy protocols were approved at both the UCSD and Buenos Aires sites.

The transgastric cholecystectomy work was performed at the Legacy Health System. The endolumenal work was performed at UCSD and the Legacy Health System as part of the institutional review board (IRB)-approved multicenter Restorative Obesity Surgery Endolumenal (ROSE) registry. The inclusion and exclusion criteria for the various procedures and approaches are denoted in Tables 1, 2. All the patients seen in the general surgery clinic were evaluated in terms of the inclusion and exclusion criteria for entrance to this study. If they were found to be a eligible, the risks and benefits of all the procedures were thoroughly discussed, and the decision was made to proceed.

All translumenal procedures used a 5-mm laparoscopic port at the beginning of the operation to confirm the safety of the entrance into the abdominal cavity. A prospective database was maintained for all cases, with an emphasis on safety, procedural success, operating time, and the postoperative pain score scaled from 1 to 10 . For the endolumenal revision cases, an outpatient upper endoscopy was performed before each procedure to document pouch and stoma dilation as a cause of weight regain and to confirm adequate working space for use of the IOP.

Surgical technique

\section{Transgastric appendectomy}

General anesthesia was confirmed, and a Foley catheter was placed. The patient's abdomen was prepped and draped in standard sterile fashion. A 5-mm Visiport (Ethicon, Cincinnati, OH USA) was placed in the umbilicus using a Hassan technique. The abdomen was insufflated to $14 \mathrm{mmHg}$, and a diagnostic laparoscopy was performed. The decision then was made to proceed with transgastric appendectomy. Upper endoscopy was performed, and the Transport device was advanced into the stomach. Using an endoscopic needleknife, a small gastrotomy was made in the anterior gastric wall. All these procedures were performed under direct laparoscopic vision.

An endoscopic esophageal balloon was advanced over a guidewire through the gastrotomy site, and the 20-mm esophageal balloon (Boston Scientific, Natick, MA, USA) was filled to dilate the gastrostomy site. Once this was achieved, the Transport device was advanced into the peritoneal cavity. The Transport device was maneuvered into position for optimal positioning to proceed with the appendectomy.

Through the working channels of the Transport device, an endoscopic grasper (Olympus America, Inc.) was used to grasp the appendix and retract it to the anterior abdominal wall. Next, dissection of the mesoappendix was performed using a combination of endoscopic graspers placed through the transport lumen and various rigid laparoscopic instruments placed through the umbilical port. The mesoappendix was divided using a harmonic scalpel (Ethicon). Endoscopic endoloops (Olympus America, Inc.) then were deployed thought the Transport device and placed at the base of the appendix.

Once the base was secured, the appendix was transected using endoscopic shears. Next, the appendix was grasped with an endoscopic grasper on the endoloops and removed from the abdominal cavity through the gastrotomy site and then through the mouth. Abdominal inspection was performed endoscopically, and the area was irrigated using suction irrigation through the umbilical port. The gastrotomy then was closed using three passes of the g-Prox device. The abdomen was desufflated, followed by removal of the 5-mm port and the endoscope. The skin was approximated on the abdomen, and the patient was awakened from general anesthesia.

\section{Transvaginal cholecystectomy}

General anesthesia was confirmed, and a nasogastric tube was placed. The patient then was placed in the dorsal lithotomy position. The abdomen and vagina were prepped in standard sterile fashion. A Foley catheter was placed on the operative field in sterile fashion. A 5-mm Visiport was placed at the umbilicus using an open Hassan technique. 
The abdomen was insufflated to $14 \mathrm{mmHg}$, and a diagnostic laparoscopy was performed. Uterine dilating sounds were placed, and a uterine retractor was used. Using a 15mm dilating trocar (Applied Medical, Rancho Santa Margarita, CA, USA), the posterior cul-de-sac was accessed. All this was performed under direct laparoscopic view.

Next, the Transport was advanced into the abdomen and positioned facing the right upper quadrant. An endoscopic grasper was placed through the Transport to grasp the dome of the gallbladder and then retracted cephlad. This was performed under laparoscopic and endoscopic view. Endoscopic loop cautery was used for careful dissection of the peritoneal layer from the gallbladder. The ultrasonic dissector then was used from the 5-mm trocar to remove the peritoneum further and delineate the anatomy.

An articulating Maryland dissector was introduced through the 5-mm port to create the cystohepatic window. Another endoscopic grasper was used to grasp the infundibulum of the gallbladder for lateral retraction to provide a clear critical view. Once this was accomplished, a 5-mm clip applier (Ethicon) was used from the umbilical port to clip the cystic duct. Endoscopic shears then were used through the Transport to transect the cystic duct. An endoscopic snare was used with electrocautery to dissect the gallbladder from the liver bed. Hemostasis was ensured. The gallbladder was grasped using an endoscopic snare and removed from the abdomen via the vaginotomy. The abdomen was desufflated, and the vaginotomy was closed primarily using 2-0 Vicryl suture. The skin was closed, and the patient was awakened from anesthesia.

\section{Transgastric cholecystectomy}

General anesthesia was induced, and a Foley catheter was placed. The patient's abdomen was prepped and draped in standard sterile fashion. A 3-mm Visiport (Ethicon) was placed in the umbilicus using a Hassan technique. The abdomen was insufflated to $14 \mathrm{mmHg}$, and diagnostic laparoscopy was performed. The decision then was made to proceed with transgastric cholecystectomy. Upper endoscopy was performed, and the Transport device was advanced into the stomach. Using an endoscopic needleknife, a small gastrotomy was made in the anterior gastric wall. All this was performed under direct laparoscopic vision.

An endoscopic esophageal balloon was advanced over a guidewire through the gastrotomy site, and the balloon was filled to dilate the gastrostomy site. Once this was achieved, the Transport device was advanced into the peritoneal cavity. All this was performed under direct laparoscopic view.

Next, the Transport was advanced into the abdomen and positioned facing the right upper quadrant. An endoscopic grasper was placed through the Transport to grasp the dome of the gallbladder and then retracted cephlad. This was performed under laparoscopic and endoscopic view.

Endoscopic hook cautery was used to dissect the peritoneal layer carefully from the gallbladder. The ultrasonic dissector then was used from the 5-mm trocar to remove the peritoneum further and to delineate the anatomy.

An articulating Maryland dissector was introduced through the 5-mm port to create the cystohepatic window. Another endoscopic grasper was used to grasp the infundibulum of the gallbladder for lateral retraction to provide a clear critical view. Endoscopic shears then were used through the Transport to transect the cystic duct. An endoscopic snare was used with electrocautery to dissect the gallbladder from the liver bed. Hemostasis was ensured, and a final peritonosocpy was performed. The gallbladder was grasped using an endoscopic snare and removed from the abdomen via the gastrotomy. The gastrotomy then was closed using three passes of the g-Prox device. The abdomen was desufflated, followed by removal of both the 3-mm port and the endoscope. The skin was approximated on the abdomen, and the patient was awakened from general anesthesia.

\section{Transumbilical cholecystectomy}

General anesthesia was induced, and a nasogastric tube was placed, followed by placement of a Foley catheter. An incision was made in the umbilicus, and the Transport was placed into the abdominal cavity using a Hassan technique under direct vision with the endoscope in the Transport. The abdomen then was insufflated through the Transport to $14 \mathrm{mmHg}$. Diagnostic laparoscopy was performed, and the decision was made to proceed with cholecystectomy.

The gallbladder dome was grasped using an endoscopic grasper and retracted cephlad. Another grasper was introduced, and the infundibulum was retracted laterally. An endoscopic snare then was introduced through the Transport and connected to electrocautery for removal of the peritoneal layer.

Once the peritoneum had been dissected from the cystic duct, an endoscopic dissector was introduced and the cystohypatic window created. The cystic duct was grasped using an endoscopic grasper, then transected using endoscopic shears. Either end of the cystic duct then was ligated and secured using endoscopic endoloops. The gallbladder was removed from the liver bed using the endoscopic snare, then grabbed with the snare and removed transumbilically.

A final peritonoscopy was performed from the endoscope to ensure hemostasis, and the abdomen was desufflated. Both the fascia and skin were closed. The Foley catheter and nasogastric tube were removed, and the patient was awakened from general anesthesia. 


\section{Endolumenal pouch and stoma reduction}

General anesthesia was induced. The stomach was intubated with the endoscope, after which the pouch and stoma sizes were measured. The g-Prox instrumentation was advanced through the Transport into the stomach. The g-Lix device was used to retract the gastric wall toward the endoscopist, and a bite was taken with the g-Prox.

A polyester anchor basket was deployed on either side of the tissue undergoing plication. Once the baskets had been deployed, they were cinched together, tightening the stitch. This sequence was repeated multiple times, first around the stoma and then around the pouch. This plicated the tissue in both places, thus reducing the stoma and pouch sizes. Once an acceptable size reduction had occurred, the stomach was inspected for hemostasis, and the device was removed. The patient then was awakened from anesthesia.

\section{Results}

A total of 29 cases were attempted for three different procedures using four separate access routes. Procedural success using the Transport through a single access point was obtained in 27 of 29 cases. Two of the endolumenal revision procedures were not completed due to technical or anatomic factors that impaired proper execution and use of instrumentation. Blood loss was minimal in all cases and less than $100 \mathrm{ml}$.

The mean operative time was $269.5 \pm 45.3 \mathrm{~min}$ for transgastric cholecystectomy, $86 \pm 8.2 \mathrm{~min}$ for transvaginal cholecystectomy, 120 min for transumbilical cholecystectomy, $273.5 \pm 54.4 \mathrm{~min}$ for transgastric appendectomy, and $79 \pm 39.8 \mathrm{~min}$ for endolumenal bypass revision. Of the 10 NOTES cases, 5 required only one transabdominal trocar (Table 2). To ensure safety and optimize retraction, additional trocars were needed in half of the NOTES cases. One transgastric cholecystectomy patient had prolonged abdominal pain necessitating an exploratory laparoscopy and upper endoscopy. An etiology for the pain was not elucidated, and the patient was discharged the next morning.

Two of the endolumenal stoma and pouch revision patients remained overnight, whereas the remainder were discharged the same day. Of the 29 patients undergoing these procedures with IOP, 27 were discharged within $24 \mathrm{~h}$.

The pain score (chosen from a scale of 1-10) at discharge was a mean of $0.44 \pm 0.8$ for endolumenal procedures, $2.25 \pm 1.3$ for transgasatric cholecystectomy, 0 for transvaginal cholecystectomy, 3 for transumbilical cholecystectomy, and $2.5 \pm 0.7$ for transgastric appendectomies. No common bile duct injuries, wound infections, or intrabdominal abscesses were noted. At the 1-week followup visit, all the patients were without complaint, with wellhealing incisions.

\section{Discussion}

The NOTES working group white paper stressed the need for new equipment to address the drive toward incisionless surgery [3]. In 2005, Pasricha et al. [4] reported on the development of this first-generation incisionless platform that combined the steerability and flexibility of an endoscope with the stability, force transmission, and triangulation of instruments familiar to laparoscopic surgeons. Accompanying preclinical work by Seaman et al. [5] demonstrated the potential of the platform's anchor delivery system to create durable transmural tissue folds reliably. Clinical use of this access platform followed quickly, with new applications and approaches being used.

Endolumenal

Swain et al. [6] reported on the development, testing, and preclinical use of the IOP system for intragastric endosurgery. Herron et al. [7], showed the feasibility of using the system for restoring the gastric pouch and gastrojejeunostomy stoma size after gastric bypass as an incisionless alternative to traditional revisional surgery.

With adequate space for the equipment, we were able to achieve substantial intraoperative gastric pouch and gastrojejeunostomy stoma reduction with use of the anchor delivery system as part of this registry work. Inadequate pouch length $(<4 \mathrm{~cm}$ from proximal stoma to the top of the pouch), impaired visualization, and faulty instrument use resulted in two unsuccessful restorative attempts. Bessler et al. [8] showed early weight loss results with clinical use of the IOP in the pilot Restorative Obesity Surgery Endolumenal (ROSE) study.

\section{Transgastric}

Transgastric surgery has many limitations and challenges. Ability to enter the peritoneal cavity safely, attempts to operate in a retroflexed position with changes in the horizon, a stable operating platform for attempts to use instrumentation made for endoscopic operation in the abdomen, and a reliable gastric closure have remained the most elusive. Our experience has allowed us to begin addressing some of these limitations.

Swanstrom et al. [9] first reported on the potential of the IOP for a transgastric approach. In 2008, Legacy Health System and Northwestern University partnered in the early 
successful clinical use of the IOP for five transgastric cholecystectomies. Sclabas et al. [10] initially reported on the potential application of the g-Prox grasper and anchor delivery system for gastrotomy closure using a transgastric approach. Swanstrom et al. [11] followed with images demonstrating use of the g-Prox for closure of the transgastric cholecystectomy gastrotomy in four clinical cases.

Horgan et al. [12] was the first to report on the successful clinical use of the IOP for a transgastric appendectomy. These first case reports showed the distinct advantage of having a stable operating platform in a transgastric setting. The Transport allows the surgeon to place the endoscope in the right upper quadrant without retroflexing the scope. Consequently, the surgeon can have freedom of movement from the tip of the Transport and the endoscope, thus allowing for a more natural view during surgery and maintenance of their horizon. The IOP also allows the surgical team the ability to switch their endoscopic instruments easily without withdrawing the endoscope from the patient or losing positioning in the abdominal cavity.

To ensure a safe entrance to the gastrotomy and finally its closure, a 5-mm laparoscopic port, or hybrid procedure, is used in all transgastric cases. From the laparoscope, the surgeon can ensure that the needleknife does not injure surrounding organs or tissue. Finally, the g-Prox proved to be a possible gastric closure device and was successful in all transgastric cases.

\section{Transvaginal}

Many of the first NOTES cases were managed via a transvaginal access. This approach has been more popular than the transgastric approach due to the ease of vaginotomy closure. Although the closure is easier, transvaginal surgery has many challenging aspects. Some of the challenges include difficult access with obese patients, difficulty maintaining a steady image with the horizon in the correct direction, pelvic adhesions hindering a safe "total NOTES" entrance, pelvic brim hindering the use of rigid instrumentation, and lack of surgical instruments that can be used through the endoscope.

In our experience, the IOP allows for a steady image once the platform is positioned in the right upper quadrant. Leaving the Transport in place allows for smooth transition of instrumentation without loss of access to the abdominal cavity. Adding further working channels through the Transport allows surgeons more freedom to work within the abdominal cavity because they have further options during the operation. Once access is obtained, the IOP allows for control of the access site so that in obese patients, it is never lost. Maneuvering into the platform over the pelvic brim and into position allows us to use our endoscopic instrumentation without running into the pelvic brim. Finally, we have used a hybrid NOTES approach in all cases to ensure safe entrance into the abdominal cavity.

\section{Transumbilical}

Single-site laparoscopy, although familiar to laparoscopists, is fraught with its own disadvantages and challenges. Having all instrumentation through the same access point in the patient's abdominal wall creates a challenge for steady visualization, triangulation, space within which to operate, and solid organ removal through one small incision.

Using the IOP through a single-site access, the umbilicus, we were able to overcome some of the aforementioned disadvantages. The ability of the IOP to maneuver flexibly within the peritoneal cavity and be locked in position has distinct advantages over current single-port technology. Once the Transport is in position, the flexible endoscope is introduced and can be manipulated freely within the abdomen. With the longer endoscope, the surgeon's assistant is removed from the immediate operative field in which the surgeon's hands are working. We are able to clean the endoscope without removing it from the abdomen, and the visual field remains stable and clear without constant interference from the working instrumentation. Articulating instrumentation aids in triangulation, although further advances in this field will be helpful. Finally, we are able to create one facial defect large enough for removal of the gallbladder without difficulty. The facial edges are approximated and the skin closed with a pleasing cosmetic result.

\section{Conclusion}

We have used the IOP to perform operations in four separate access points. The Transport allowed for a stable operating platform, stable vision of the operative field, a direct conduit into the peritoneal cavity, and easy instrument exchange. This platform has proved itself to be safe and access neutral, enabling the use of one system for natural orifice and single-site-access approaches.

Disclosures Santiago Horgan is a consultant for USGI. Garth Jacobsen is a consultant for USGI. Lee. Swanstrom is a consultant for USGI. Kari Thompson, Mark Talamini, Alberto Ferreres, Georg Spaun, and John Cullen have no conflicts of interest or financial ties to disclose.

Open Access This article is distributed under the terms of the Creative Commons Attribution Noncommercial License which permits any noncommercial use, distribution, and reproduction in any medium, provided the original author(s) and source are credited. 


\section{References}

1. Cottam DR, Nguyen NT, Eid GM, Schauer PR (2005) The impact of laparoacopy on bariatric surgery. Surg Endosc 19:621-627

2. Keus F, deJong JA, Gooszen HG, vanLaarhoven CJ (2006) Laparoscopic versus open cholecystectomy for patients with symptomatic cholelithiasis. Cochrane Database Syst Rev 4: CD006231

3. SAGES ASGE (2006) ASGE/SAGES working group on natural orifice translumenal endoscopic surgery white paper October 2005. Gastrointest Endosc 63:199-203

4. Pasricha P, Kozarek R, Swain P, Swanstrom LL, Raju G, Gross S, Saadat V, Rothe C, Birkett D (2005) Development of a nextgeneration therapeutic endoscope. Gastrointest Endosc 61: AB106-AB108

5. Seaman DL, Gostout CJ, de la Mora Levy JG, Knipschield MA (2006) Tissue anchors for transmural gut-wall apposition. Gastrointest Endosc 64:577-581

6. Swain P, Kozarek R, Pasricha PJ et al (2005) The development and testing of a new multichannel, shape-locking guide with tip and midbody articulation for intragastric endosurgery. Gastrointest Endosc 61:AB183

7. Herron DM, Birkett DH, Thompson CC, Bessler M, Swanstrom LL (2008): Gastric bypass pouch and stoma reduction using a transoral endoscopic anchor placement system: a feasibility study. Surg Endosc 22:1093-1099. Epub 20 November 2007

8. Bessler M, Stevens PD, Milone L, Swanstrom LL (2008) An endosurgical operating system: human experience in endolumenal and NOTES procedures. SAGES 2008 podium presentation, Philadelphia, PN, April 11, 2008

9. Swanstrom LL, Kozarek R, Pasricha PJ et al (2005) Development of a new access device for transgastric surgery. J Gastrointest Surg 9:1129-1136; discussion 1136-1137

10. Sclabas G, Swain P, Swanstrom LL (2006) Endolumenal therapy for gastrotomy closure in natural orifice transenteric surgery (NOTES) Surg Innov 13:23-30

11. Swanstrom LL, Hungness E, Whiteford ME, Soper NJ (2008) Use of the g-Prox tissue approximation device for gastrotomy closure in human NOTES procedures. SAGES 2008 poster presentation, Philadelphia, PN

12. Horgan S, Cullen JP, Talamini MA, Mintz Y, Ferreres A, Jacobsen GR, Sandler B, Bosia J, Savides T, Easter DW, Savu MK, Ramamoorthy SL, Whitcomb E, Agarwal S, Lukacz E, Dominguez G, Ferraina P (2009) Surg Endosc. doi:10.1007/s00464-009-0428-0. epub 03 April 2009 